Prediction rule

\section{Physician practice and PECARN rule outperform CATCH and CHALICE rules based on the detection of traumatic brain injury as defined by PECARN}

10.1136/ebmed-2014-110090

Franz E Babl, ${ }^{1,2,3}$ Silvia Bressan ${ }^{1,4}$

${ }^{1}$ Murdoch Children's Research Institute, Australia; ${ }^{2}$ Royal Children's Hospital, Australia; ${ }^{3}$ University of Melbourne, Melbourne, Australia; ${ }^{4}$ University of Padova, Italy

Correspondence to: Professor Franz E Babl, Emergency Department, Royal Children's Hospital, Flemington Road, Parkville, VIC 3052, Australia; franz.babl@rch.org.au

Commentary on: Easter JS, Bakes K, Dhaliwal J, et al. Comparison of PECARN, CATCH, and CHALICE rules for children with minor head injury: a prospective cohort study. Ann Emerg Med 2014;64:145-52.e5.

\section{Context}

The recognition of significant traumatic intracranial injuries is important and cranial CT is the gold standard for their diagnosis. However, CT bears risks associated with ionising radiation-induced malignancies, in particular in children. Three high-quality clinical decision rules (CDR) have been developed to assist with decision-making on whether or not to use a cranial CT scan in children who sustain a trauma to the head ${ }^{1}$ : the Canadian Assessment of Tomography for Childhood Head injury $(\mathrm{CATCH})^{2}$ the Children's Head injury Algorithm for the prediction of Important Clinical Events (CHALICE) ${ }^{3}$ and the rule developed by the Pediatric Emergency Care Applied Research Network (PECARN). ${ }^{4}$ They differ significantly in their predictor variables and suggested course of action. They have been derived using different outcomes, inclusion and exclusion criteria and have focused on different severities of head injuries. Only PECARN has been validated outside the derivation population. ${ }^{5}$ It is unclear which rule is the best for clinicians to use.

\section{Methods}

This single centre prospective observational study compared the diagnostic accuracy of the CATCH, CHALICE and PECARN CDRs as well as physician judgement. The study included children younger than 18 years with GCS $\geq 13$ presenting within $24 \mathrm{~h}$ of a blunt head injury. Patients with brain tumours, ventricular shunts and bleeding disorders were excluded. Main outcome measure was the presence of a clinically important traumatic brain injury (ciTBI) as defined by PECARN. ${ }^{4}$ Physicians recorded predictor variables of each CDR before results of imaging were available. In addition, physician practice and a likelihood of perceived risk of ciTBI was recorded. For patients not undergoing CT, a combination of medical record review, follow-up phone calls and trauma registries was used to determine if the primary outcome had occurred. Each CDR was considered positive when at least one of the predictor variables was present.

\section{Findings}

In 1009 children, 21 had ciTBI. All were identified by the PECARN rule and physician practice. Ranked sensitivities were as follows: physician practice and PECARN 100\% (95\% CI 84\% to 100\%), physician estimates 95\% (95\% CI $76 \%$ to $100 \%$ ), CATCH $91 \%$ (95\% CI 70\% to 99\%) and CHALICE $84 \%$ (95\% CI 60\% to 97\%). Ranked specificities were: CHALICE 85\% (95\% CI $82 \%$ to $87 \%$ ), physician estimates 68\% (95\% CI 65\% to 71\%), PECARN 62\% (95\% CI 59\% to 66\%), physician practice 50\% (95\% CI 47\% to 53\%), and CATCH 44\% (95\% CI 41\% to 47\%). Secondary outcomes included need for neurosurgical intervention with sensitivities of 100\% for PECARN and physician practice and 75\% for CATCH and CHALICE.

\section{Commentary}

This paper is the first to compare the accuracy of the three highest quality CDRs for head injuries in children. While it sets out to evaluate the diagnostic accuracy of the CDRs and physician practice, the primary outcome was defined based on PECARN alone. It found that PECARN and physician practice were the only approaches that identified all ciTBI with PECARN slightly more specific. This would imply that PECARN was the best of the CDRs studied. The authors acknowledge the limitations of a small sample size with only 21 ciTBI and 4 patients requiring neurosurgical intervention resulting in large and overlapping 95\% CIs.

While the use of only one outcome definition is an understandable solution to the problem of comparing three different rules, it introduces important methodological limitations based on differences in the definitions of outcomes. PECARN alone, for example, includes hospital admission $>2$ nights for head injury in its definition of ciTBI.

While CHALICE and CATCH were developed to identify children at higher risk of intracranial injuries, the PECARN rule was designed to identify children at very low risk of ciTBI. So 'meeting the criteria' for CHALICE and CATCH implies having at least one positive predictor (ie, CT scan required), while for PECARN this translates into having none of the predictors (ie, no CT scan required). This could have been made more explicit in the Easter paper for a correct interpretation of the rules in clinical practice.

The results should not be interpreted as external validation of the three rules. This would have required the application of the specific inclusion and exclusion criteria and the outcome definitions used for each rule. CHALICE applies to a broad population of head injuries of any severity, PECARN was developed for minor head injuries only and CATCH focused on a group of patients with specific signs or symptoms. A strict application of the inclusion criteria for CATCH in particular narrows the applicability of the CDR to only about a quarter of emergency department head injury presentations as presented in web appendix E4 and shown elsewhere. ${ }^{6}$ Similarly, CHALICE was developed for children under 16 years. The exclusion of a single 17-year-old in the study would have increased the sensitivity of neurosurgical intervention as outcome for CHALICE from 75\% to $100 \%$.

While ideally repeated in a larger data set, this pragmatic study provides a useful guide for clinicians. In settings with experienced clinicians, physicians seem to be able to recognise children with possible ciTBI. In other settings, PECARN appears to provide a good guide.

\section{Contributors FEB and SB were involved in writing the commentary.}

Competing interests None.

Provenance and peer review Commissioned; internally peer reviewed.

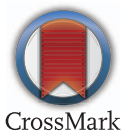




\section{References}

1. Lyttle MD, Crowe L, Oakley E, et al. Comparing CATCH, CHALICE, and PECARN Clinical Decision Rules for Paediatric Head Injuries. Emerg Med J 2012;29:785-94.

2. Osmond MH, Klassen TP, Wells GA, et al. CATCH: a clinical decision rule for the use of computed tomography in children with minor head injury. CMAJ 2010;182:341-8.

3. Dunning J, Daly JP, Lomas JP, et al. Derivation of the children's head injury algorithm for the prediction of important clinical events decision rule for head injury in children. Arch Dis Child 2006;91:885-91.

4. Kuppermann N, Holmes JF, Daya PS, et al. Identification of children at very low risk of clinically-important brain injuries after head trauma: a prospective cohort study. Lancet 2009;374:1160-70.

5. Schonfeld D, Bressan S, Da Dalt L, et al. Pediatric Emergency Care Applied Research Network head injury clinical prediction rules are reliable in practice. Arch Dis Child 2014;99:427-31.

6. Lyttle MD, Cheek JA, Blackburn C, et al. Applicability of the CATCH, CHALICE and PECARN paediatric head injury clinical decision rules: pilot data from a single Australian centre. Emerg Med J 2013;30:790-4. 